HD-THEP-99-25

\title{
Bosonic Preheating in Left-Right-Symmetric SUSY GUTs
}

\author{
R. Micha円, M.G. Schmidt巴 \\ Institut für Theoretische Physik, Philosophenweg 16, D-69120 Heidelberg, \\ Germany
}

\begin{abstract}
We investigate the possibility of a bosonic preheating in the simplest model of supersymmetric Hybridinflation (F-term inflation), which was considered first by Dvali et al. Here the inflationary superpotential is of the O'Raifertaigh-Witten type. The end of inflation is related to a non-thermal phase transition, which in the context of left-right symmetric models lowers the rank of the gauge group. Using the homogeneous classical field ansatz for the appearing condensates, our results indicate that the parametric creation of bosonic particles does not occure in the model under consideration.
\end{abstract}

\footnotetext{
${ }^{1}$ r.micha@thphys.uni-heidelberg.de

${ }^{2}$ m.g.schmidt@thphys.uni-heidelberg.de
} 


\section{Introduction}

The recent success in detecting neutrino masses has caused a renaissance of the idea of grand unification. In left-right-symmetric models, such as $S U(3)_{c} \times$ $S U(2)_{L} \times S U(2)_{R} \times U(1)_{(B-L)}$ or $S O(10)$, massive Majorana neutrinos arise naturally. As the difference of baryon- and lepton number is a gauged symmetry in these models, there could exist interesting mechanisms for the creation of the corresponding asymmetries. On the other hand, supersymmetry not only provides the right mass scale for the heavy neutrinos, but also gives the possibility of a inflationary potential in the GUT-Higgs-Sektor. Then it is possible to construct a cosmological model based on a consistent supersymmetric grand unified theory, which can be judged by both, its cosmological and its particle theoretical features. In this paper we will concentrate on an O'Raifertaigh-Witten model, which by the autors of [1] was shown to be a realisation of Linde's Hybrid Inflation scenario [2]. The superpotential can serve as a part of the Higgs sector of a left-right-symmetric model with global or local supersymmetry. The phase of inflation and the formation of density fluctuation in this model have been studied in [1, 3]. Since a successful cosmological model needs a very effective mechanism for particle production after inflation, here we investigate the possibility of a bosonic preheating in the inflationary potential under consideration. As preheating possibly allows for the creation of superheavy particles, it is a very interesting scenario not only in the context of lepto- and baryogenesis but also for the creation of dark matter. Focusing on qualitative insights rather than quantitative accuracy, we tried to work with a minimum of numerical expense. Our investigation was inspired by those in [4, 5], where preheating in the original version of Hybrid Inflation was studied. Our numerical results, which originally were presented in the context of an investigation of inflationary supersymmetric $S O(10)$-models in [6], are very similiar to those in a recent paper, based on a version of a NMSSM [7]. But, as shall be seen, we disagree with the physical interpretation of the results given there. In contrast to [7], we do not see any evidence for bosonic preheating in supersymmetric hybrid inflation.

\section{Supersymmetric Hybridinflation: the Basic Scenario}

A common way to achieve an inflationary scenario from a supersymmetric theory, is to use the simplest O'Raifertaigh-Witten model for spontaneous symmetry breakdown [1]. It is given by the superpotential

$$
W=X\left(\kappa \bar{C} C-\mu^{2}\right),
$$

where the couplings are protected by either a continouus or a discrete $R$-symmetry, which could descend from a string theory. The superfield $C=\left(\hat{a}_{C}, \hat{\psi}_{C}\right)$ is a rep- 
resentation of the Lie-algebra $A_{G}$ of the Lie-group $\mathrm{G}$, and $\bar{C}=\left(\hat{a}_{\bar{C}}, \hat{\psi}_{\bar{C}}\right)$ is the conjugate representation. Here the $\hat{a}$ 's represent the scalar component while the $\hat{\psi}$ 's are the spinors. The Superfield $X=\left(\hat{a}_{X}, \hat{\psi}_{X}\right)$ is an $A_{G^{-}}$Singlet. The mass-scale $\mu$ could be caused by a string compactification. In the case, that $G$ represents a gauge symmetry we have the tree-level scalar potential

$$
\begin{aligned}
V^{(0)} & =\left|F_{X}\right|^{2}+\left|F_{C}\right|^{2}+\left|F_{\bar{C}}\right|^{2}+\frac{1}{2} \sum_{r=1}^{\operatorname{dim}(G)}\left|D_{r}\right|^{2} \\
& =\kappa^{2}\left|a_{X} a_{\bar{C}}\right|^{2}+\kappa^{2}\left|a_{X} a_{C}\right|^{2}+\left|\kappa a_{\bar{C}} a_{C}-\mu^{2}\right|^{2}+\frac{1}{2} \sum_{r=1}^{\operatorname{dim}(G)}\left|D_{r}\right|^{2} .
\end{aligned}
$$

Here $a_{i}$ represents the vacuum expectation value (vev) of the quantum field $\hat{a}_{i}$. Then $V^{(0)}$ is minimized for $\arg a_{\bar{C}}+\arg a_{C}=0$ and it is independent of $\arg a_{\bar{C}}+\arg a_{X}$ and $\arg a_{C}+\arg a_{X}$. Supersymmetry requires the $D$-terms to vanish identically for each single generator of the group $G$. If $G$ represents a product group including an $U(1)$-factor, this means that $\left|a_{C}\right|=\left|a_{\bar{C}}\right|$.

In the following we will be interested only in the direction of the $A_{G}$-multiplets $a_{C}$ and $a_{\bar{C}}$, which aquire a $\mathrm{G}$ breaking vev. Therefore they will be denoted by the same names. Now, concentrating on the $D=0$-direction, there exists a certain $R$-transformation [8] which brings the scalar components $a_{i}$ to the real axis. The corresponding canonically normalized scalarfields $\phi, \sigma$ and couplings $\lambda, g$ are given by [8]:

$$
\begin{aligned}
\sigma & :=2 a_{C}=2 a_{\bar{C}} \\
\phi & :=\sqrt{2} a_{X} \\
\lambda & :=\frac{\kappa^{2}}{4} \\
g & :=\frac{\kappa}{\sqrt{2}} \\
M & :=\sqrt{\kappa} \mu,
\end{aligned}
$$

where typically $g^{2}=2 \lambda$. Using this conventions the scalar effective potential in terms of the fields $\phi$ and $\sigma$ reads

$$
V(\phi, \sigma)=\frac{1}{4 \lambda}\left(M^{2}-\lambda \sigma^{2}\right)^{2}+\frac{1}{2} g^{2} \phi^{2} \sigma^{2}+V^{(1)}(\phi)
$$

which resembles very much Linde's original model of Hybrid Inflation [2]. Here $V^{(1)}(\phi, \sigma)$ represents the loop corrections to the tree level potential, which vanish for the supersymmetric case. For $\sigma=0, \phi>M / g=: \phi_{c}$ the gauge symmetry remains conserved while supersymmetry is broken in the sector of the gauge singlet $X$ inducing loop-corrections $V^{(1)}(\phi, \sigma)$. Including 1-loop-corrections the 
effective potential in this regime is given by [1]

$$
\begin{aligned}
V\left(\phi>\phi_{c}\right)= & \frac{M^{4}}{4 \lambda}\left(1+\frac{\lambda}{4 \pi^{2}}\left[\ln \frac{2 \lambda \phi^{2}}{M^{2}}+\left(\frac{2 \lambda \phi^{2}}{M^{2}}-1\right)^{2} \ln \left(1-\frac{M^{2}}{2 \lambda \phi^{2}}\right)+\right.\right. \\
& \left.\left.+\left(\frac{2 \lambda \phi^{2}}{M^{2}}+1\right)^{2} \ln \left(1+\frac{M^{2}}{2 \lambda \phi^{2}}\right)+\ln \frac{M^{2}}{\Lambda^{2}}\right]\right),
\end{aligned}
$$

where $\Lambda$ is a renormalization scale. Since soft susy breaking terms lead to scalar masses of $\mathcal{O}(\mathrm{TeV})$, such terms are neglegible compared to the GUT-scale mass parameter $M$. For a large range of the parameters $\kappa$ and $\mu$ the potential above satisfies the Slow Roll conditions for inflation. Here inflation is caused by the "cosmological constant" $\frac{M^{4}}{4 \lambda}$. The scalar gauge singlet is the only degree of freedom, which then has a nonvanishing vev $\phi$. This vev very soon is dominated by the zero momentum Fourier mode and it forms a condensate with a very small extension in momentum space. Referring to [9] it can be approximated by a homogeneous classical scalar field. For this reason we will call $\phi$ the inflaton and identify it with the zero momentum mode. The slow roll regime of the singlet is a typical aspect of the superpotential (2.1) and has become popular as effect of "a sliding field" outside the context of inflation [10].

The end of inflation is connected to the end of the gauge symmetric phase: as soon as $\phi$ reaches the critical point $\phi_{c}$ the effective mass $m_{\sigma}^{2}=-M^{2}+g^{2} \phi^{2}$ of the Higgs-field vanishes. The classical equations of motions alone cannot tell us something about a phase transition, since the derivative $V\left(\phi_{c}, \sigma=0\right){ }_{\sigma}$ vanishes identically and the vev of $\hat{\sigma}$ remains zero. But, following the argumentation of Garcia-Bellido and Linde [4], as $\phi$ slides towards zero, quantum fluctuations around this vev will get tachyonic masses stimulating an exponential growth of modes, whose momenta are smaller than the effective mass, $k<\left|m_{\sigma}\right|$, where $k$ means the comoving momentum. The modes with $k>\left|m_{\sigma}\right|$ will not grow at all. The result is a spontaneous breakdown of the gauge symmetry caused by the inhomogenous distribution of the field $\hat{\sigma}$ with $\langle\hat{\sigma}\rangle=0$. Inflation ends typically near this phase transition.

We now want to understand, if the behaviour of the Higgs field $\hat{\sigma}$ can be approximated by an homogeneous classical field. As pointed out in [4] the distribution of the Higgs field would be homogeneous on scales $l \sim\left|m_{\sigma}\right|^{-1}$ or even somewhat greater, if $m_{\sigma}$ was constant in time. However, we will see soon that this mass is oscillatory. This changes the range and the mechanism of amplification of the "tachyonic modes". Due to this fact, the distribution $\sigma_{k}(t)$ in momentum space will be even more localized around the zero momentum mode, such that the spatial distribution will be homogeneous even on a scale $l$ which is somewhat greater than $\left|m_{\sigma}\right|^{-1}$. At least at such scales $\hat{\sigma}(t, \vec{x})$ also can be effectively described by a classical homogenous scalar field $\sigma(t)$, i.e. as the zero momentum mode of such a classical field, which rolls down from the critical point 
to the supersymmetric minimum, where $\sigma=M / \sqrt{\lambda}$. We will call $\sigma(t)$ the Higgscondensate. The range of validity for this ansatz will become clearer during our numerical investigation.

As pointed out in [3] local supersymmetry modifies this scenario because of the non-renormalizable terms, whose influence on the inflation depends on the value of the coupling constant $\kappa$. But in most of these cases the end of inflation is dominated by the renormalizable terms in the potential. Thus, nonrenormalizable couplings will not contribute after the phase transition and will be ignored in the following investigation.

\section{Evolution of the Background}

After the phase transition both of the fields $\hat{\phi}(t, \vec{x})$ and $\hat{\sigma}(t, \vec{x})$ form condensates, which essentially can be described by the two homogeneous classical fields $\phi(t)$ and $\sigma(t)$. Free Higgs or singlet particles will be identified with quantum fluctuations around these condensates. In this section we concentrate on the evolution of the background and we neglect the effect of the quantum fluctuations. For reasons of simplification we use a "M-rescaling" to natural variables:

$$
\begin{aligned}
t \rightarrow y & :=M t \\
\vec{x} \rightarrow \vec{\xi} & :=M \vec{x} \\
\vec{k} \rightarrow \vec{K} & :=\frac{\vec{k}}{M} \\
\phi \rightarrow f & :=\frac{\phi g}{M}=\frac{\phi}{\phi_{c}} \\
\sigma \rightarrow s & :=\frac{\sigma \sqrt{\lambda}}{M}=\frac{\sigma}{\sigma_{c}} .
\end{aligned}
$$

Here the comoving momenta $\vec{K}$ and the scale factor $a(y)$ are normalized in such a way that $a\left(y_{c}\right)=1$ is fullfilled, where $y_{c}$ means the M-rescaled time at the phase transition.

Neglecting the sub-dominant loop corrections, which certainly will appear, as long as the supersymmetric minimum is not reached, the equations of motion after the phase transition read:

$$
\begin{gathered}
\left(\frac{d^{2}}{d y^{2}}+3 h(y) \frac{d}{d y}+2 s^{2}(y)\right) f(y)=0 \\
\left(\frac{d^{2}}{d y^{2}}+3 h(y) \frac{d}{d y}+\left(-1+f^{2}(y)+s^{2}(y)\right)\right) s(y)=0
\end{gathered}
$$

where $h$ is the M-rescaled Hubble Parameter, $h:=\left(\frac{d}{d y} a\right) / a$, with

$$
h^{2}(y)=\frac{2 \pi}{3} \frac{M^{2}}{\lambda M_{p l}^{2}}\left[2 \dot{f}^{2}(y)+\dot{s}^{2}(y)+\left(1-s^{2}(y)\right)^{2}+2 f^{2}(y) s^{2}(y)\right] .
$$




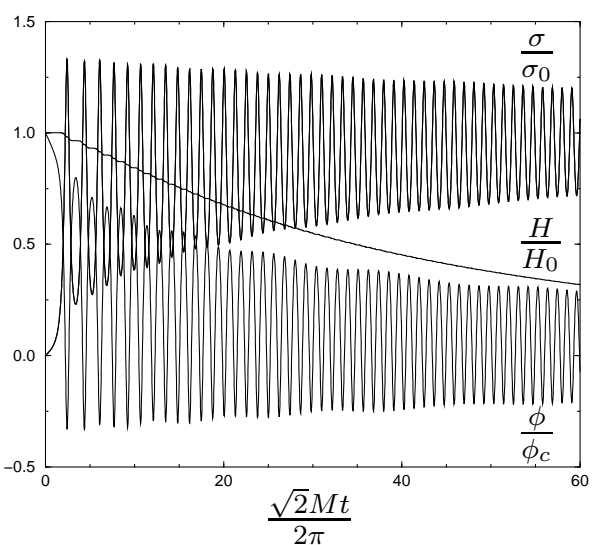

Figure 3.1: Evolution of the background

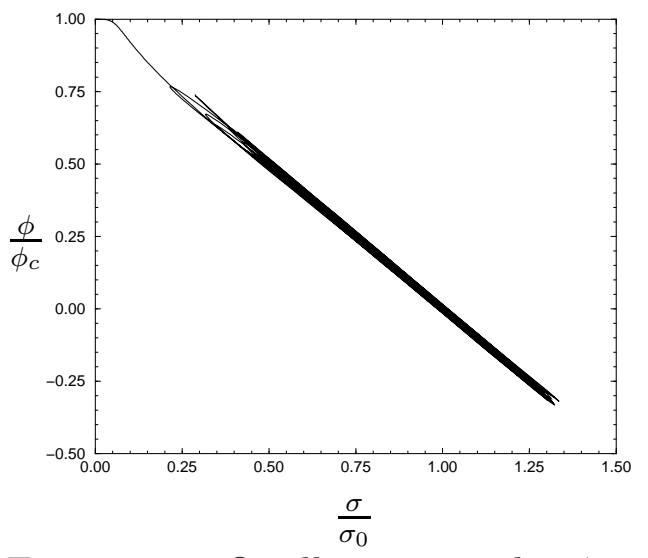

Figure 3.2: Oscillations in the $\phi-\sigma$ plane

Important qualitative insights then are possible without any detailed calculation: Both of the classical fields, $f=\frac{\phi}{\phi_{c}}$ and $s=\frac{\sigma}{\sigma_{0}}$, effectively oscillate with the same frequency, approximately given by $\omega=\sqrt{2}$, around the supersymmetric minimum, $\phi=0, \sigma=M / \sqrt{\lambda}$. For energetic reasons the phase difference amounts to $\pi / 2$. Coming from the critical point $(\phi=M / g, \sigma=0)$, depending on the initial values the dynamics in the $\phi-\sigma$-plane should tend to be nearly one dimensional. Since in our case the initial time derivates of the fields should be very small slow roll values the trajectory should be near to a straight line. Another interesting point, obvious without any calculation, is that the dynamics of the post-inflationary system, measured in its natural time and lengthscale $M^{-1}$, only depends on the scale of the phase transition, $M / \sqrt{\lambda}$. As the M-rescaled classical fields $f$ and $s$ and their derivatives, take maximal values of $\mathcal{O}(1)$ effectively, this scale has influence on the damping but not on any other aspects of the background oscillations: the smaller the scale is, the longer the system swings correponding to its natural timescale $M^{-1}$. Thus, qualitative results of one breaking scale will also appear at another breaking scale.

This discussion is confirmed by our numerical integration of the system of equations, as shown in figures 3.1 and 3.2. The parameters used in this and all of the following investigations are given by

$$
\begin{aligned}
\lambda & =0.625 \times 10^{-3} \\
g^{2} & =0.125 \times 10^{-2} \\
M & =0.350 \times 10^{15} \\
\frac{M}{\sqrt{\lambda}} & =1.40 \times 10^{16},
\end{aligned}
$$

which corresponds to a phase transition near to the SUSY-GUT scale in a supersymmetric $S O(10)$ model in [6]. The solutions are essentially damped oscillations around the sypersymmetric minimum, with the predicted phase. The dynamics 


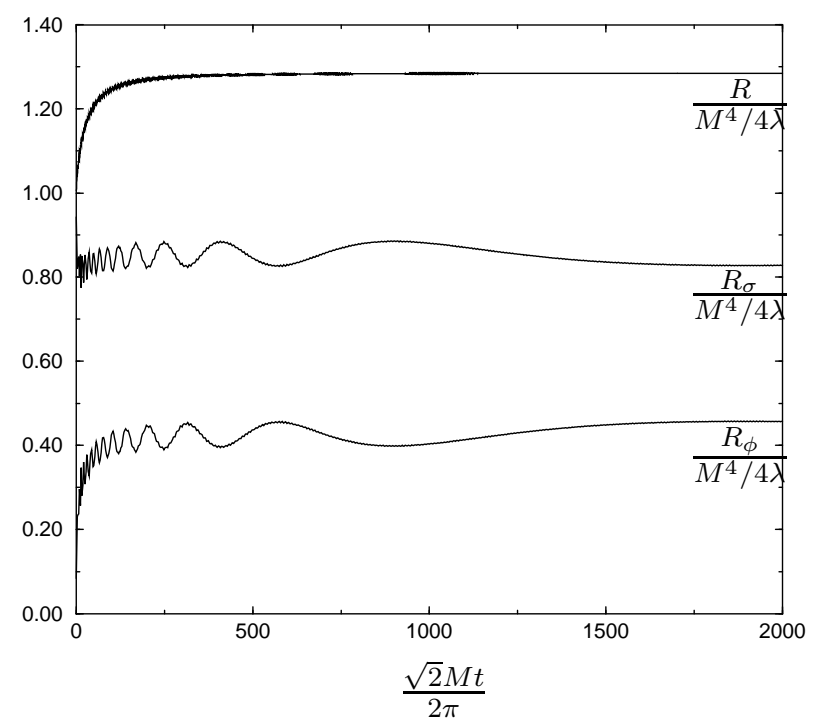

Figure 3.3: energy densities of the background

in the $f$-s-plane is practically one dimensonal and can be restricted to the straight line for very small initial values of the time derivatives of the fields. Anharmonic behaviour is caused by the interaction of the classical fields and by the fact, that the Higgs field has a negative mass contribution from the tachyonic mass.

Our result for the supersymmetric case $\left(g^{2}=2 \lambda\right)$ is quite different to the case with $g^{2}=\lambda$, which was considered in [4], where the classical fields oscillate in a rather chaotic way. But it essentially equals the result in [7].

Now we consider the comoving energy densities after the phase transition:

$$
\begin{aligned}
R & :=\rho a^{3}, \\
R_{\phi} & :=\rho_{\phi} a^{3}, \\
R_{\sigma} & :=\rho_{\sigma} a^{3}
\end{aligned}
$$

Here approximately $\rho=M^{4} / 4 \lambda$ is the system's total energy density at the phase transition. As the Universe passes a phase of matter domination after inflation, such that $\rho \sim a^{-3}$, the product $\rho a^{3}$ will be constant. This is what our numerical investigations show in figure 3.3. After the phase transition $R$ is growing for a short time, up to the value of $1.24 M^{4} / 4 \lambda$ and then remains practically constant. It takes the background about 120 oscillations until it swings in such a ,harmonic" way, that the universe behaves matter dominated. Since we were interested in the effective distribution of energy between both of the swinging classical fields, in the calculation of $R_{\phi}$ and $R_{\sigma}$ we integrated out the short time dynamics $\left(t \sim \mathcal{O}\left(M^{-1}\right)\right)$ by averaging over the the background oscillations. Here we respected the fact, that the frequency of the oscillations is slightly varying with time. The plot shows, how energy is exchanged between the classical fields - one reason for anharmonic behaviour in figure 3.1. After about 2000 oscillations the 
energy distribution between the oscillation of the inflaton and Higgs-condensate is approximately $1 / 3: 2 / 3$. Then the classical fields essentially are decoupled oscillators.

\section{Parametric Excitation of Quantum Fluctua- tions}

Our investigation of the quantum fluctuations uses the M-rescaling introduced in section 3. We studied the mode equations following from the Heisenberg expansion of a quantum field $\hat{\chi}$, which stands for the M-rescaled quantum fluctuations $\delta \hat{s}(y, \vec{\xi})$ and $\delta \hat{f}(y, \vec{\xi})$ around the classical fields $s(y)$ and $f(y)$ :

$$
\begin{aligned}
\hat{\chi}(y, \vec{x}) & =\frac{1}{(2 \pi)^{3}} \int d^{3} p\left(\hat{b}_{p} X_{p}(y) e^{-i a \vec{\xi} \vec{p}}+\hat{b}_{p}^{+} X_{p}^{*}(y) e^{i a \vec{\xi} \vec{p}}\right), \\
& =\frac{1}{(2 \pi)^{3} a^{3 / 2}(y)} \int d^{3} K\left(\hat{b}_{K} X_{K}(y) e^{-i \vec{\xi} \vec{K}}+\hat{b}_{K}^{+} X_{K}^{*}(y) e^{i \vec{\xi} \vec{K}}\right),
\end{aligned}
$$

where $\vec{p}=\vec{K} / a$ is the M-rescaled physical momentum corresponding to the Mrescaled commoving momentum $\vec{K}$. The operators $\hat{b}_{p}^{(+)}$satisfy the commutation relation:

$$
\left[\hat{b}_{p}, \hat{b}_{p^{\prime}}^{+}\right]=(2 \pi)^{3} \delta\left(\vec{p}-\vec{p}^{\prime}\right)
$$

Using the comoving momenta $K=|\vec{K}|=|\vec{p}| a$ for notation, this reads

$$
\frac{1}{a(y)^{3}}\left[\hat{b}_{K}, \hat{b}_{K^{\prime}}^{+}\right]=(2 \pi)^{3} \delta\left(\vec{K}-\vec{K}^{\prime}\right) .
$$

Then the occupation number is given by

$$
n_{K}=\frac{\omega_{K}}{2}\left(\frac{\left|\dot{X}_{K}\right|^{2}}{\omega_{K}^{2}}+\left|X_{K}\right|^{2}\right)-\frac{1}{2},
$$

and the particle density reads:

$$
n_{\chi}(y)=\int \frac{d^{3} K}{(2 \pi a)^{3}} n_{K} .
$$

Neglecting higher order terms in the quantum fluctuations, the mode equations read:

$$
\begin{gathered}
\left(\frac{d^{2}}{d y^{2}}+\left(\frac{K^{2}}{a^{2}}+2 s^{2}-\frac{3}{2} \frac{\ddot{a} a+\dot{a}^{2} / 2}{a^{2}}\right)\right) \delta f_{K}(y)=0 \\
\left(\frac{d^{2}}{d y^{2}}+\left(\frac{K^{2}}{a^{2}}+3 s^{2}+f^{2}-1-\frac{3}{2} \frac{\ddot{a} a+\dot{a}^{2} / 2}{a^{2}}\right)\right) \delta s_{K}(y)=0,
\end{gathered}
$$




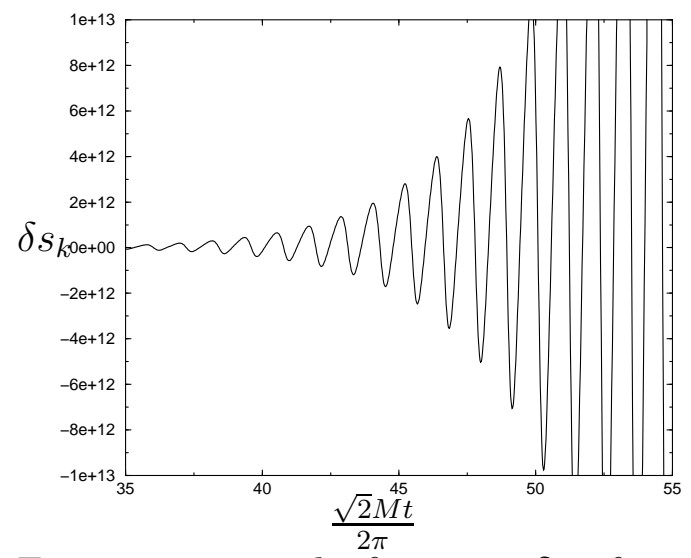

Figure 4.1: mode function $\delta s_{k}$ for $k=M / 10$

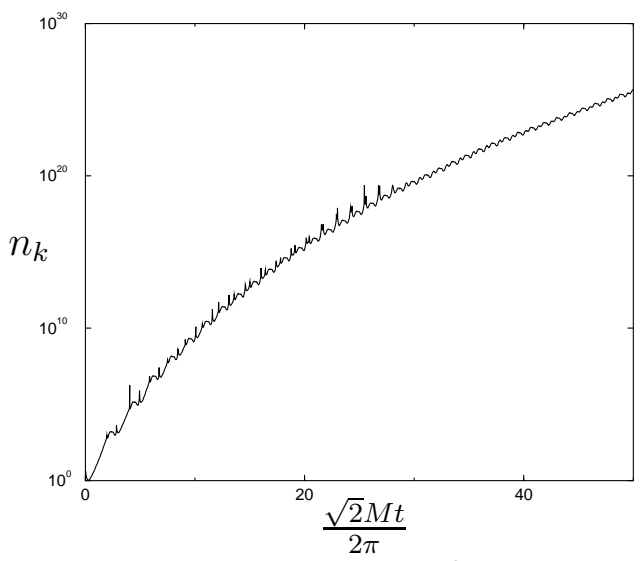

Figure 4.2: resonance of the mode with $k=M / 10$

As numerical calculations show, very soon $(3 / 2)\left(\ddot{a} a+\dot{a}^{2} / 2\right) /\left(a^{2}\right) \sim 0$ is fullfilled within the numerical accuracy and this term can be neglected. This corresponds to a matter dominated universe, where typically $\ddot{a} a=-\dot{a}^{2} / 2$. We performed a numerical integration of the full set of differential equations. The values of $K$, for which these investigations were done, were selected by a pre-investigation using the well known approximation by the Mathieu equation [11.

Before turning over to our numerical results, it is sensible first to consider the problem of particle interpretation. As clearly pointed out before, both of the classical fields, $\phi$ and $\sigma$, are approximate representations of the quantum fields $\hat{\phi}(t, \vec{x})$ and $\hat{\sigma}(t, \vec{x})$ in the low momentum zone. Although we identified these classical fields with their zero-momentum modes in principle they have an extension in momentum space. The edge of the condensates in momentum space will be situated at a momentum which is somewhat smaller than the mass of the corresponding field. In this sense the use of a homogeneous classical field is similiar to a limitation of the ,resolution" in an optical investigation. But if this is true, interpreting the quantum fluctuations around these classical fields as free particles in presence of a homogeneous background is justified only for those modes, whose momenta are well above the edges of the condensates. This important point was apparently not taken into consideration by the authors of [7].

As in the last section, we performed numerical calculations of the full system of differential equations mentioned above. We did not find any parametric amplification of the Higgs-fluctuations with $K \gtrsim 1 / 3$. For $K \lesssim 1 / 3$ there exists an amplification which can be described as a mixing of both effects: mainly parametric resonance and partly tachyonic mass. In the well known picture of the Mathieu equation,

$$
\left(\frac{d^{2}}{d z^{2}}+A_{k}-2 q \cos (2 z)\right) \chi_{k}=0
$$


which is applicable restricting only to some few oscillations, this resonance starts inside the first resonance band $\left(1-q \lesssim A_{k} \lesssim 1+q\right)$ with a resonance parameter $q \sim 0.9$, which corresponds to a strong narrow resonance regime. The amplification of these modes is essentially independent of the momentum and is given by the solution for $K=0$. Furthermore, these modes lie in a range of momenta, which we estimated in section 2 to have non-vanishing fourier modes of the Higgs-condensate: Because of this we do not consider these soft modes to be free particles. We think, that using the homogeneous classical field ansatz it does not make sense to study Higgs fluctuations with such momenta. Our result only tells us someting about the extension of the distribution of $\hat{\sigma}$ which was described in section 2 .

The picture in the case of inflaton fluctuations is similiar. Here a tachyonic mass is missing and the effect of the resonance is much weaker. But there is no amplification of modes with $K \gtrsim 1 / 10$ and similiar to the above case, the observed weak parametric amplification for modes with $K \lesssim 1 / 10$ cannot be intepreted as a creation of free particles.

In order to learn more about the space/momentum dependence of the condensates one could calculate the mode equation of the fourier modes $s_{K}$ and $f_{K}$ of the condensates. Since the original equations of motions are non-linear, the mode equations then are be rather complicated integro-differential equations. A first step of simplification would be to let their masses only be given by the zero momentum modes of the condensates. As can be checked easily, the mode equations for $f_{K}$ are exactly the same as for $\delta f_{K}$. Those for $s_{K}$ will look a little bit different from those for $\delta s_{k}$ but will have similiar resonance parameters in the Mathieu approximation. Therefore, from the investigation that we did for the quantum fluctuations one can conclude that there will be a similiar resonance effect in a similiar range of momenta for the modes $s_{K}$ and $f_{K}$.

This shows that after the phase transition the inflaton condensate slightly looses its homogenity, while the Higgs condensate is non-homogeneous from the very beginning. However, at a physical scale which is somewhat bigger than $a M^{-1}$ they are essentially homogeneous. Quantum fluctuations around these condensates should have monumenta which are bigger than the inverse scale of homogenity, in order to have a particle interpretation.

Until now our investigations did not consider the effect of backreaction of the quantum fluctuations on the classical fields, which is very important in the case of a preheating with a very efficient particle production. As pointed out in [11], the main effect of the backreaction is to lower the resonance parameters in the picture of the Mathieu equation. This means, that appearing resonances will become weaker and will last for a shorter time. Then, in our case, the general picture is not changed. The extension of the condensates in momentum space probably will be smaller, such that they will be even "more homogeneous" than without backreaction. But there will be no possibility of having a parametric amplification of fluctuations, which were not amplified also without backreaction. 


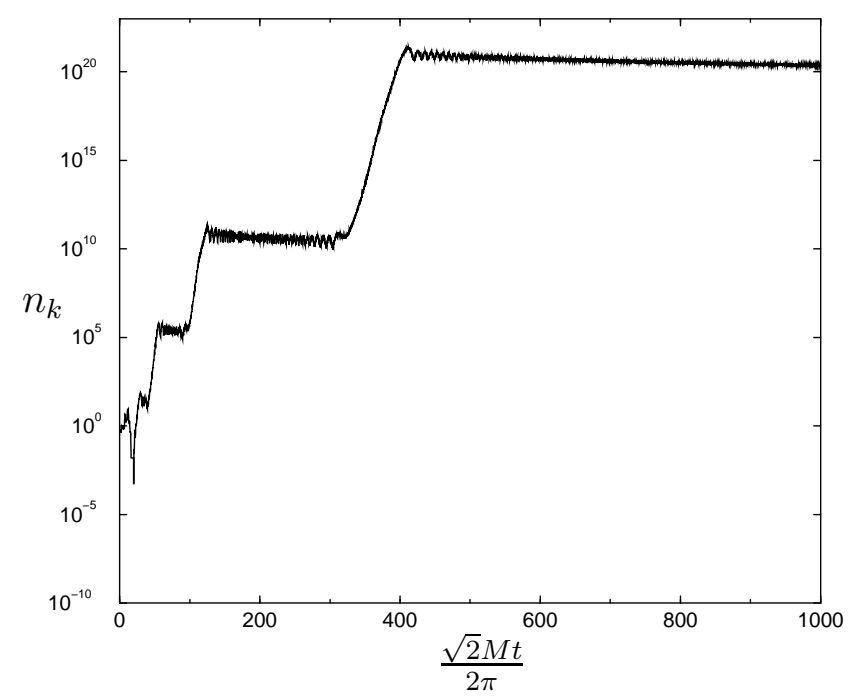

Figure 5.1: parametric excitation of the $k=M$-mode of a scalar field $\hat{\chi}$

So we conclude that there does not exist a creation of free Higgs- or singletparticles by parametric amplification. This corresponds to the result in [4] for the different case of $\lambda=g^{2}$.

\section{Parametric Excitations of External Fields}

Although we did not find a preheating of the quantum fluctuations, there could be a parametric creation of an external scalar quantum field $\hat{\chi}$, that couples to the Higgs sector via

$$
\frac{1}{2} h_{1}^{2} \phi^{2} \hat{\chi}^{2}+\frac{1}{2} h_{2}^{2} \sigma^{2} \hat{\chi}^{2}
$$

Then the equations of motions will be modified in an obvious way. The mode equation for the modes $\chi_{K}(t)$ reads

$$
\left(\frac{d^{2}}{d y^{2}}+\left(\frac{K^{2}}{a^{2}(t)}+\frac{h_{1}^{2}}{g^{2}} f^{2}+\frac{h_{2}^{2}}{\lambda} s^{2}\right)\right) \chi_{K}(y, \vec{\xi})=0
$$

where again $\ddot{a} a=-\dot{a}^{2} / 2$ was assumed.

We used the same methods as before in our numerical investigation. Our result is very similiar to the result in [4] for $g^{2}=\lambda$. We found a strong resonance only for the case without coupling to the Higgs-condensate, $h_{2} \simeq 0$ (see figure 5.1). But this situation is impossible, if we use the superpotential (2.1). The Fterms always lead to $h_{2} \neq 0$ if $h_{1} \neq 0$ and vice versa. As can be shown very easily [6], in this model there does not exist any coupling allowed by supersymmetry and leading to a bosonic parametric resonance. This result, as far as we can see, buries the hope for the existence of a bosonic preheating. 


\section{Consequences for Left-Right Symmetric Mod- els}

In left-right-symmetric models the phase transition that leads to the gauge symmetry of the Standard Model, necessarily lowers the rank of the Lie group. The only renormalizable superpotential with this feature has the structure

$$
W=X \bar{C} C+\text { polynomial in } X,
$$

where $X$ is a gauge singlet and $\bar{C}, C$ are appropriate spinor representations of the left-right-symmetric gauge group. It is an interesting question, if, using the superpotential (2.1), there could be a natural embedding of inflation into the context of a right-left-symmetric model such as $S O(10)$ [6] or $S U(3)_{c} \times S U(2)_{L} \times$ $S U(2)_{R} \times U(1)_{(B-L)}$, which leads to a senseful cosmological model.

In minimal $S O(10)$-models [12, 13] for example $C$ should be a 16-dimensional spinor representation, the scalar component of which aquires a GUT-scale vev in the $S U(5)$-singlet direction during the phase transition. Since the $S O(10)$ (more precisely: Spin(10)) is a simple and simply connected Lie group, breaking it down to the SM-gauge group will lead to unwanted monopoles. They appear in a first phase transition, when a 45 dimensional Higgs representation aquires a suitable vev to break $\operatorname{Spin}(10)$ down to the left-right-symmetric group $G_{L R}:=$ $S U(3)_{c} \times S U(2)_{L} \times S U(2)_{R} \times U(1)_{(B-L)}$. The inflaton then dilutes the unwanted remnants and ends in a phase transition which breakes the remaining gauge symmetry to the standard model group. This picture remains the same in a pure $G_{L R}$-Model, $C$ being a $S U(2)_{R} \times U(1)_{(B-L)}$-Higgs-doublet.

Our investigations indicate, that in such models free bosonic particles cannot be created by the effect of parametric resonance after inflation. The parametric amplification in the ,low momentum zone" of the quantum fluctuations, which appears in our numerical investigation, cannot be interpreted as a production of free particles. In this area not only the dynamics of the mode functions are essentially independent of the momentum but also the validity of the homogeneous classical field ansatz is doubtful. In our view, these numerical results perhaps can tell us something about the extension of the condensates in momentum space but shurely show us the limitation of the homogeneous classical field ansatz.

Supposing that bosonic preheating does not take place, a next step would be to study the possibility of fermionic preheating for this superpotential, which recently was shown to be possibly very efficient [14]. Since we deal with a supersymmetric theory, there is no reason for neglecting the fermionic superpartners. Also a parametric creation of Majorana neutrinos could appear, which should be very interesting in the context of leptogenesis. In this context it also would be nessecary to rule out the creation of the helicity-1/2-gravitinos by non-perturbative

effects, which recently was shown to be of possible danger for cosmological models which involve supersymmetry [15]. If fermionic preheating turned out not to be 
efficient, particle production in left-right-symmetric models could be caused only by perturbative effects. Following [16] then still the production of super heavy matter could be possible. But, as we work with two condensates, both of them would have to decay very efficiently, in order to be not in conflict with standard cosmology. Considering the gravitino constraint on the reheating temperature of the Universe, this would mean strong limitations to possible couplings to other fields. Concluding, there is still some work to do, until the particle creation after inflation in this very simple supersymmetric model will be fully investigated.

\section{Acknowledgment}

We would like to thank S. J. Huber and P. John for useful discussions. This work was supported in part by the TMR network Finite Temperature Phase Transitions in Particle Physics, EU contract no. ERBFMRXCT97-0122. 


\section{References}

[1] G. Dvali, Q. Shafi, R. Schaefer, Phys. Rev. Lett. 73 (1994) 1886-1889 [hep$\mathrm{ph} / 9406319]$

[2] A. Linde, Phys. Rev. D52 (1994) 748-754 [astro-ph/9307002v3]

[3] A. Linde, A. Riotto, Phys. Rev. D56 (1997) 1841-1844 [hep-ph/9703209]

[4] J. Garcia-Bellido, A. Linde, Phys. Rev. D 57 (1998) 6075-6088 [hep$\mathrm{ph} / 9711360]$

[5] J. Garcia-Bellido, [hep-ph/9804205]

[6] R. Micha, Preheating in der supersymmetrischen SO(10)-Vereinheitlichung, Diplomarbeit, Heidelberg, February 1999

[7] M. Bastero-Gil, S. F. King, J. Sanderson, [hep-ph/9904315]

[8] G. Lazarides, Q. Shafi, Phys. Lett. B 372 (1996) 20-24 [hep-ph/9510275]

[9] A. Linde, Particle Physics and Inflationary Cosmology, Harwood Academic Publishers, Chur, 1990

[10] N. Dragon, U. Ellwanger, M. G. Schmidt, Progr. Part. Nucl. Phys. 18 (1987) 1

[11] L. Kofman, P. B. Greene, A. Linde, A. A. Starobinsky, Phys. Rev. D 56 (1997) 6175-6192 [hep-ph/9705347]; L. Kofman, A. Linde, A. A. Starobinsky, Phys. Rev. D56 (1997) 3258-3295 [hep-ph/9704452]

[12] K. S. Babu, S. M. Barr, Phys. Rev. D51 (1995) 2463-2470

[13] S. M. Barr, S. Raby, Phys. Rev. Lett. 79 (1997) 4748-4751 [hep-ph/9705366 v2]

[14] J. Baacke, K. Heitmann, C. Pätzold, Phys. Rev. D58 (1998) 125013 [hepph/9806205]; P. B. Greene, L. Kofman, Phys. Lett. B448 (1999) 6-12 [hep-ph/9807339]; G. F. Giudice, M. Peloso, A. Riotto, I. Tkachev, [hep$\mathrm{ph} / 9905242]$

[15] R. Kallosh, L. Kofman, A. Linde, A. van Proeyten, [hep-th/9907206 ]; G. F. Giudice, I. Tkachev, A. Riotto, [hep-ph/9907510]

[16] D. J. H. Chung, E. W. Kolb, A. Riotto, [hep-ph/9809453 ] 